\title{
A NOTE ON BESSEL FUNCTION DUAL INTEGRAL EQUATION WITH WEIGHT FUNCTION
}

\author{
B.N. MANDAL \\ Department of Mathematics \\ University of Calcutta \\ 92 Acharya Prafulla Chandra Road \\ Calcutta - 700009 \\ INDIA
}

(Received February 16, 1987 and in revised form May 15, 1987)

ABSTRACT. An elementary procedure based on Sonine's integrals has been used to reduce dual integral equations with Bessel functions of different orders as kernels and an arbitrary weight function to a Fredholm integral equation of the second kind. The result obtained here encompasses many results concerning dual integral equations with Bessel functions as kernels known in the literature.

KEYS WORDS AND PHRASES. Dual integral equations, Bessel function, Sonine's integrals, Hankel inversion, Fredholm integral equation.

1980 AMS SUBJECT CLASSIFICATION CODE. $45 \mathrm{~F}$.

1. INTRODUCTION.

In a recent paper Nasim [1] considered the following class of dual integral equations involving Bessel function of first kind of different orders as kernels and arbitrary weight function

$$
\left.\begin{array}{l}
\int_{0}^{\infty} t^{-2 \alpha} J_{\nu}(x t)[1+w(t)] \phi(t) d t=f(x), 0<x<1, \\
\int_{0}^{\infty} t^{-2 \beta} J_{\mu}(x t) \phi(t) d t=g(x), x>1 .
\end{array}\right\}
$$

An operational procedure based on exploiting the properties of Mellin transform is used in [1] to reduce (1.1) to a single integral equation involving Bessel function as kernel. By Hankel inversion this was then reduced to a Fredholm integral equation of second kind. However, the general result in [1] was obtained under the assumptions that $|\alpha-\beta|<\frac{\mu-\nu}{2}+1$ and $\nu \geq 0$, although the second assumption was not clearly stated. This is necessary to make $\lambda>-1$ (where $\lambda=\frac{\mu+\nu}{2}-\alpha+\beta$ ) for the Hankel inversion to be valid. It is not clear whether this operational procedure involving Mellin transform, can be used if these restrictions on the parameters $\mu, \nu, \alpha, \beta$ are not satisfied. This has motivated us to reexamine the dual integral equations (1.1) for arbitrary values of the parameters $\mu, \nu, \alpha, \beta$. 
Earlier Noble [2] used the 'multiplying-factor method' based on Sonine's integrals to a class of dual integral equations with Bessel functions of same order $(\mu=\nu)$ as kernels to a Fredholm integral equation of second kind. Schmeltzer and Lewin [3] also used this multiplying-factor method to reduce another class of dual integral equations involving first kind Bessel functions of different orders and a particular weight function to dual integral equations with trigonometric sine function as kernels in closed form by the function-theoretic method. Later Ross [4] gave a simplified method of construction of explicit solution to the class of dual integral equations considered in [3]. Also Rose and de Hoog [5] considered another class of dual integral equations with some particular weight functions for explicit solution. These dual integral equations arose when standard integral transform approach was used to solve some mixed boundary value problems were solved by using a complex variable technique. The explicit solution to the dual integral equations were then deduced from the solutions to the boundary value problems. Except for these special class of dual integral equations considered in $[3,4,5]$, the general class of dual integral equations (1.1) does not appear to admit of explicit solution. References to most of the works on different dual integral equations with Bessel function kernels can be found in $[1,2,4,6]$.

While in [2] dual integral equations with first kind Bessel functions of the same order as kernels were considered, in the present paper as we11 as in [1], integral equations with Bessel functions of different orders as kernels are considered. Here we employ the multiplying-factor method of Noble [2] to reduce (1.1) to a single integral equation with Bessel function as kernel. Invoking Hankel inversion, this single integral equation is then reduced to a Fredholm integral equation of second kind. The result is obtained for all arbitrary values of the parameters $\mu, U, \alpha, \beta$. From this result many known results concerning dual integral equations with Bessel function kernels can be deduced. As special cases, three sets of values of the parameters are considered. The general result obtained here then reduces to the known results for these special cases.

2. REDUCTION TO A FREDHOLM INTEGRAL EQUATION OF SECOND KIND.

We assume that the parameters $\mu, \nu, \alpha, \beta$ appearing in (1.1) are most general. By using the formulae

$$
\begin{aligned}
& \left(\frac{1}{x} \frac{d}{d x}\right)^{p}\left[x^{-\mu} J_{\mu}(x t)\right]=(-1)^{p} x^{-\mu-p} t^{p} J_{\mu+p}(x t), \\
& \left(\frac{1}{x} \frac{d}{d x}\right)^{q}\left[x^{\mu} J_{\mu}(x t)\right]=x^{\mu-q} t^{q} J_{\mu-q}(x t)
\end{aligned}
$$

where $p, q$ are non negative integers, it is always possible to change the orders $\nu, \mu$ to $\nu^{\prime}, \mu^{\prime}$ respectively such that $\nu^{\prime}-1, \mu^{\prime}>-3 / 2$ (of course, $\alpha, \beta$ are then also changed) by choosing $p$ suitably. Thus without any loss of generality, we can assume that the orders $\nu, \mu$ of the Bessel functions in (1.1) satisfy the restrictions $\nu>-1, \mu>-3 / 2$. These restrictions are necessary in the analysis that follows. Multiplying each side of the first equation in $(1.1)$ by $\left(r^{2}-x^{2}\right)^{\xi} x^{u+1}$ where $\xi>-1$, integrating from 0 to $r(<1)$ and using Sonine's first integral in the form 


$$
\begin{aligned}
\int_{0}^{r}\left(r^{2}-x^{2}\right)^{\xi} & x^{\nu+1} J_{\nu}(x t) d x \\
& =2^{\xi} \quad \Gamma(\xi+1) t^{-\xi-1} r^{\xi+\nu+1} J_{\nu+\xi+1}(r t), \nu>-1, \xi>-1,
\end{aligned}
$$

we obtain

$$
\begin{aligned}
2^{\xi} \quad \Gamma(\xi+1) & r^{\xi+\nu+1} \int_{0}^{\infty} t^{-2 \alpha-\xi-1} J_{\nu+\xi+1}(r t)[1+w(t)] \phi(t) d t \\
& =\int_{0}^{r} x^{\nu+1}\left(r^{2}-x^{2}\right)^{\xi} f(x) d x, 0<r<1
\end{aligned}
$$

Similarly, multiplying each side of the second equation in (1.1) by $\left(x^{2}-r^{2}\right)^{n} x^{-\mu+1}$ where $\eta>-1$, integrating from $r(>1)$ to $\infty$ and using Sonine's second integral in the form

$$
\begin{aligned}
\int^{\infty}\left(x^{2}-r^{2}\right)^{n} x^{-\mu+1} J_{\mu}(x t) d x \\
\quad=2^{n} \Gamma(n+1) t^{-n-1} r^{-\mu+n+1} J_{\mu-n-1}(r t),-1<n<\frac{\mu}{2}-\frac{1}{4},
\end{aligned}
$$

we obtain

$$
\begin{aligned}
2^{n} \Gamma(n+1) r^{-\mu+n+1} \int_{0}^{\infty} t^{-2 \beta-n-1} J_{\mu-n-1}(r t) \phi(t) d t \\
\quad=\int_{r}^{\infty}\left(x^{2}-r^{2}\right)^{n} x^{-\mu+1} g(x) d x, 1<r<\infty .
\end{aligned}
$$

Using (2.2) (with $q$ replaced by $\ell$ and $x$ replaced by $r$ ) in (2.4), we obtain

$$
\begin{aligned}
& \int_{0}^{\infty} t^{-2 \alpha-\xi-1+\ell} J_{\nu+\xi+1-\ell}(r t)[1+w(t)] \phi(t) d t \\
& =\frac{2^{-\xi}}{\Gamma(\xi+1)} r^{-\xi-\nu-1+l}\left(\frac{1}{r} \frac{d}{d r}\right)^{\ell} \int_{0}^{r} x^{\nu+1}\left(r^{2}-x^{2}\right)^{\xi} f(x) d x, 0<r<1,(2.7)
\end{aligned}
$$

where $\ell$ is a nonnegative integer. Similarly using (2.1) (with $p$ replaced by $m$ and $x$ replaced by $r$ ) in $(2.6)$ we obtain

$$
\begin{aligned}
& \int_{0}^{\infty} t^{-2 \beta-n-1+m} J_{\mu-n-1+m}(r t) \phi(t) d t \\
& \quad=\frac{(-1)^{m} 2^{-n}}{\Gamma(n+1)} r^{\mu-n-1+m}\left(\frac{1}{r} \frac{d}{d r}\right)^{m} \int_{0}^{\infty}\left(x^{2}-r^{2}\right)^{n} x^{-\mu+1} g(x) d x, r>1,(2.8)
\end{aligned}
$$

where $\mathrm{m}$ is a nonnegative integer.

In (2.7) and (2.8) we now equate the powers of $t$ and the orders of the Bessel functions. This gives two equations to determine $\xi, n$ as

$$
\begin{aligned}
& -2 \alpha-\xi-1+\ell=-2 \beta-\eta-1+\mathrm{m}, \\
& u+\xi+1-\ell=\mu-\eta-1+\mathrm{m} .
\end{aligned}
$$

Thus we obtain 


$$
\begin{aligned}
& \xi=\frac{\mu-\nu}{2}+\beta-\alpha-1+\ell, \\
& \eta=\frac{\mu-\nu}{2}+\alpha-\beta-1+m .
\end{aligned}
$$

The requirements that $\xi$ and $\eta$ must be greater than -1 (cf (2.3) and (2.5)) can be satisfied by choosing the nonnegative integers $\ell$ and $m$ appropriately. Thus we obtain the single integral equation

$$
\begin{aligned}
& \int_{0}^{\infty} t^{-\gamma} J_{\lambda}(r t) \phi(t) d t \\
& \quad= \begin{cases}F(r)-\int_{G(r)}^{\infty} t^{-\gamma} J_{\lambda}(r t) w(t) \phi(t) d t, & 0<r<1, \\
0 & r>1\end{cases}
\end{aligned}
$$

where

$$
\left.\begin{array}{l}
\lambda=\frac{\mu+\nu}{2}+\beta-\alpha, \\
\gamma=\frac{\mu-\nu}{2}+\alpha+\beta
\end{array}\right\}
$$

and $F(r)$ and $G(r)$ are given by the right sides in (2.7) and (2.8) respectively. Now in the single integral equation (2.10) we require $\lambda>-1$ for Hankel inversion. In general, this requirement may not be satisfied. To overcome this difficulty, we use (2.1) (with $p$ replaced by $s$ and $x$ replaced by $r$ ) in (2.10) to obtain

$$
\begin{aligned}
& \int_{0}^{\infty} t^{-\gamma+s} J_{\lambda+s}(r t) \phi(t) d t
\end{aligned}
$$

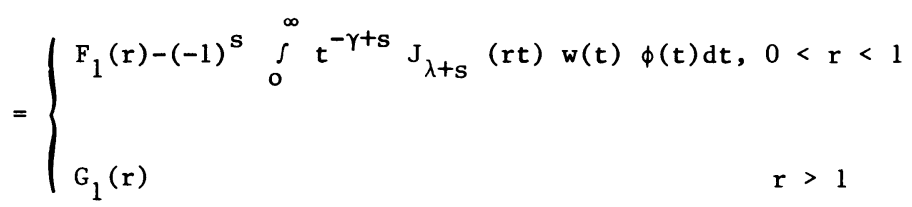

where

$$
\begin{aligned}
& F_{1}(r)=(-1)^{s} r^{\lambda+s}\left(\frac{1}{r} \frac{d}{d r}\right)^{s}\left[r^{-\lambda} F(r)\right], \\
& G_{1}(r)=(-1)^{s} r^{\lambda+s}\left(\frac{1}{r} \frac{d}{d r}\right)^{s}\left[r^{-\lambda} G(r)\right],
\end{aligned}
$$

and $s$ is a nonnegative integer. Whatever be $\alpha, \beta$ we can always choose $\lambda+s>-1$. Then the Hankel inversion can be invoked in (2.12) to give a Fredholm integral equation of second kind in $\phi(x)$ as

$$
\begin{aligned}
\phi(x)= & x^{\gamma-s+1}\left[\int_{0}^{1} r F_{1}(r) J_{\lambda+s}(r x) d r+\int_{1}^{\infty} r G_{1}(r) J_{\lambda+s}(r x) d r\right. \\
& \left.-\int_{0}^{\infty} u^{-\gamma+s} w(u) \phi(u) \frac{u J_{\lambda+s+1}(u) J_{\lambda+s}(x)-x J_{\lambda+s+1}(x) J_{\lambda+s}(u)}{u^{2}-x^{2}} d u\right]
\end{aligned}
$$


where $\lambda, \gamma$ are given in (2.11) and $s$ is a nonnegative integer to be chosen appropriately so as to make $\lambda+s>-1$.

3. SPECIAL CASES.

3.1. $\mu=\nu, \alpha=-a / 2, \beta=0$ where $\nu>\max \left(-1, \frac{1}{2}-a\right)$ and $0<a<2$. The dual integral equations are then

$$
\left.\begin{array}{l}
\int_{0}^{\infty} t^{a}[1+w(t)] \phi(t) J_{v}(x t) d t=f(x), 0<x<1, \\
\int_{0}^{\infty} \phi(t) J_{v}(x t) d t=g(x), x>1
\end{array}\right\}
$$

These dual integral equations were considered by Noble [2]. The conditions $v>-1$ and $\mu>-3 / 2$ are satisfied automatically. Now from (2.9)

$$
\xi=-1+\frac{a}{2}+l, \quad n=-1-\frac{a}{2}+m
$$

where $\ell, m$ are nonnegative integers to be chosen such that $\xi>-1, \eta>-1$. We choose $\ell=0, m=1$ so that $\xi=-1+\frac{a}{2}>-1$ and $n=-\frac{a}{2}>-1$. Also $\lambda=\nu+\frac{a}{2}>-1, \gamma=-\frac{a}{2}$. Hence we take $s=0$ in (2.14). Then

$$
\begin{aligned}
& F_{1}(r)=F(r)=\frac{2^{1-a / 2}}{\Gamma(a / 2)} r^{-v-\frac{a}{2}} \int_{0}^{r} u^{v+1}\left(r^{2}-u^{2}\right)^{-1+a / 2} f(u) d u, \\
& G_{1}(r)=G(r)=-\frac{2^{a / 2}}{\Gamma(1-a / 2)} r^{-v+\frac{a}{2}-1} \frac{d}{d r} \int_{0}^{\infty}\left(u^{2}-r^{2}\right)^{-a / 2} u^{1-v} g(u) d u,
\end{aligned}
$$

so that the Fredholm integral equation in $\phi(x)$ is

$$
\begin{aligned}
\phi(x) & =\frac{(2 x)^{1-a / 2}}{\Gamma(a / 2)} \int_{0}^{1} r^{1-v-a / 2}\left\{\int_{0}^{r} u^{v+1}\left(r^{2}-u^{2}\right)^{-1+a / 2} f(u) d u\right\} J_{v+a / 2}(r x) d r \\
& -\frac{x^{1-a / 2} 2^{a / 2}}{\Gamma(1-a / 2)} \int_{1}^{\infty} r^{-v+a / 2} \frac{d}{d r}\left\{\int_{r}^{\infty}\left(u^{2}-r^{2}\right)^{-a / 2} u^{1-v} g(u) d u\right\} \cdot J_{v+a / 2}(r x) d x \\
-x^{1-a / 2} & \int_{0}^{\infty} u^{a / 2} w(u) d(u) \frac{u J_{v+a / 2+1}(u) J_{v+a / 2}(x)-x J_{v+a / 2+1}(x) J_{v+a / 2}(u)}{u^{2}-x^{2}} d u
\end{aligned}
$$

This result was obtained in [2] (cf.eq. (4.7)).

Putting $g(x)=0, w(u)=0$, we obtain the explicit solution of the dual integral equations

$$
\begin{aligned}
& \int_{0}^{\infty} t^{a} \phi(t) J_{v}(x t) d t=f(x), 0<x<1, \\
& \int_{0}^{\infty} \phi(t) J_{v}(x t) d t=0, x>1 \\
\text { where } \quad v & \max \left(-1, \frac{1}{2}-a\right) \text { and } 0<a<2 \text {, as } \\
\phi(x)= & \frac{(2 x)^{1-a / 2}}{\Gamma(a / 2)} \int_{0}^{1} \mathrm{r}^{a / 2+1} J_{v+a / 2}(r x)\left\{\int_{0}^{1} v^{v+1} f(r v)\left(1-v^{2}\right)^{a / 2-1} \mathrm{dv}\right\} \mathrm{dr} .
\end{aligned}
$$


This coincides with the result given in [7].

3.2. $|\alpha-\beta|<\frac{\mu-\nu}{2}+1$ and $\nu \geqq 0$. This case was considered in [1]. Then $\lambda>-1$, and we may mention here that the restriction $\nu \geq 0$ was not stated explicitly in

[1]. Unless this restriction holds good, it is not possible to use Hankel inversion with the order of the Bessel function as $\lambda$. To make $\xi>-1, \eta>-1$, we choose $\ell=1, \mathrm{~m}=1$ in (2.9). Also as $\lambda>-1$, we choose $s=0$ in (2.14) so that the dual integral equations (2.1) in this case reduce to

$$
\begin{aligned}
\phi(x) & =x^{\gamma+1}\left[\frac{2^{-\gamma+2 \alpha}}{\Gamma(\gamma-2 \alpha+1)} \int_{0}^{1} r^{-\gamma+2 \alpha-\nu} J_{\lambda}(r x) d\left\{\int_{0}^{r} u^{v+1}\left(r^{2}-u^{2}\right)^{\gamma-2 \alpha} f(u) d u\right\}\right. \\
& -\frac{2^{-\gamma+2 \beta}}{\Gamma(\gamma-2 \beta+1)} \int_{1}^{\infty} r^{\lambda} J_{\lambda}(r x) d\left\{\int_{r}^{\infty}\left(u^{2}-r^{2}\right)^{\gamma-2 \beta} u^{1-\mu} g(u) d u\right\} \\
& \left.-\int_{0}^{\infty} u^{-\gamma} w(u) \phi(u) \frac{u J_{\lambda+1}(u) J_{\lambda}(x)-x J_{\lambda+1}(x) J_{\lambda}(u)}{u^{2}-x^{2}} d u\right]
\end{aligned}
$$

This result does not seem to coincide with the general result given in [1]. However, the known results for the following two special cases given in [6] as well as in [1] are deduced from (3.5) implying that (3.5) is also correct.

When $\mu=\nu=0, \alpha=1 / 2, \beta=0,(2.11)$ gives $\lambda=-1 / 2$ and $\gamma=\frac{1}{2}$. Substituting in (3.5) we obtain after simplification

$$
\begin{aligned}
\phi(x) & =\frac{2 x}{\pi} \int_{0}^{1} \cos r x d\left\{\int_{0}^{r} \frac{u f(u)}{\left(r^{2}-u^{2}\right)^{1 / 2}} d u\right\} \\
& +\frac{2 x}{\pi} \int_{0}^{\infty} \cos r x\left\{\int_{0}^{\infty} \frac{u g(u)}{\left(u^{2}-r^{2}\right)^{1 / 2}} d u\right\} d r \\
& -\frac{x}{\pi} \int_{0}^{\infty} \frac{w(u) \phi(u)}{u}\left\{\frac{\sin (u+x)}{u+x}+\frac{\sin (u-x)}{u-x}\right\} d u .
\end{aligned}
$$

This result can also be deduced directly from (2.14) by choosing $s=0, \ell=1, \mathrm{~m}=0$. When $\mu=\nu=0, \alpha=-1 / 2, \beta=0$, the integral equation for $\phi(x)$ can similarly be deduced from (3.5). In this case $\lambda=1 / 2, \gamma=-1 / 2$. After simplification we obtain

$$
\begin{aligned}
\phi(x) & =\frac{2}{\pi} \int_{0}^{1} \sin r x\left\{\int_{0}^{r} \frac{u f(u)}{\left(r^{2}-u^{2}\right)^{1 / 2}} d u\right\} d r \\
& \left.-\frac{2}{\pi} \int_{1}^{\infty} \sin r x \int_{r}^{\infty} \frac{u g(u)}{\left(u^{2}-r^{2}\right)^{1 / 2}} d u\right\} \\
& -\frac{1}{\pi} \int_{0}^{\infty} w(u) \phi(u)\left\{\frac{\sin (u-x)}{u-x}-\frac{\sin (u+x)}{u+x}\right\} d x .
\end{aligned}
$$

(3.7) can also be deduced directly from (2.14) by choosing $\mathrm{s}=0, \ell=0, \mathrm{~m}=1$.

4. DISCUSSION.

The operational procedure based on Mellin transform to reduce the integral equations (1.1) to a Fredholm integral equation of second kind as given in [1] seems too 
complicated and lengthy compared to Noble's multiplying-factor method based on Sonine's integrals as demonstrated in this note. Further, the method outlined here can be used to attack the dual integral equations (1.1) for arbitrary values of the parameters $\nu, \mu, \alpha, \beta$ and the weight function.

ACKNOWLEDGEMENT. This work was supported in part by the UGC project No.F.8-8/85 (SR III).

\section{REFERENCES}

1. NASIM, C. On dual integral equations with Hankel kernel and arbitrary weight function, Internat. J. Math. and Math. Sci. 9 (1986), 293-300.

2. NOBLE, D. The solution of Bessel function dual integral equations by a multiplying-factor method, Proc. Camb. Phil. Soc. 59 (1963), 351-362.

3. SCHMELTZER, R.A. and LEWIN, M. Function-theoretic solution to a class of dual integral equations and application to diffraction theory, Quart. Appl. Math. $21(1964), 259-283$.

4. ROSS, R.A. An integral equation arising in diffraction theory, Wave phenomenon: modern theory and application, North Holland Math. Stud. 97 (1983), 453-459.

5. ROSE, R.F. and DE HOOG, F.R. Exact solutions of certain dual integral equations and their asymptotic properties, Q. J1. Mech. Appl. Math. 36 (1983), 420-436.

6. SNEDDON, I.N. Mixed boundary value problems in potential theory, John Wiley and Sons, New York, 1966.

7. TITCHMARSH, E.C. Introduction to the theory of Fourier integrals, Oxford University Press, $19 \overline{37 .}$ 


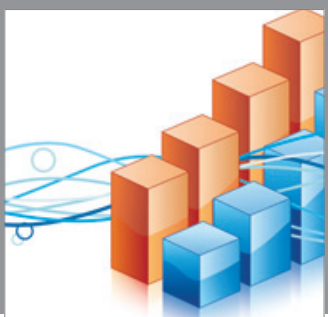

Advances in

Operations Research

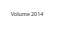

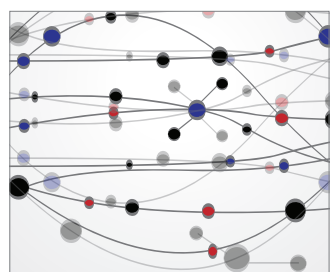

\section{The Scientific} World Journal
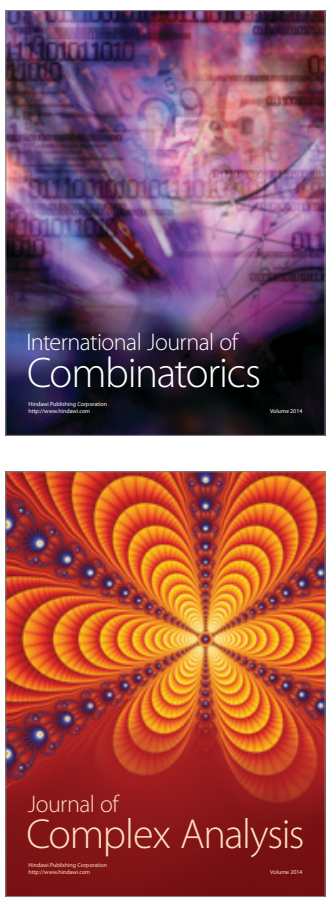

International Journal of

Mathematics and

Mathematical

Sciences
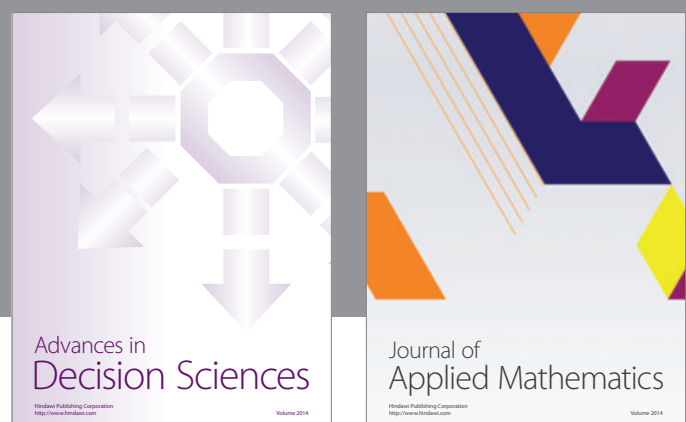

Journal of

Applied Mathematics
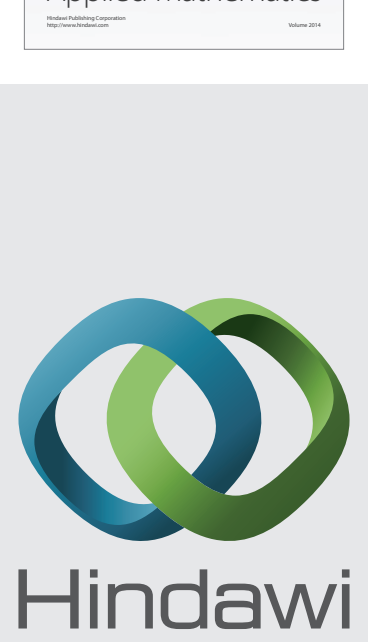

Submit your manuscripts at http://www.hindawi.com
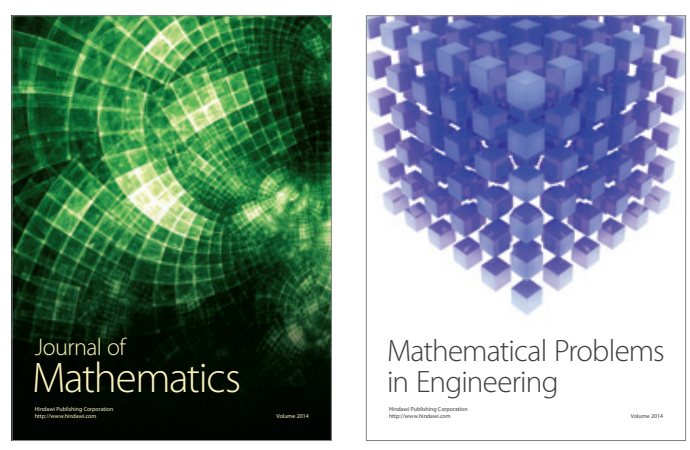

Mathematical Problems in Engineering
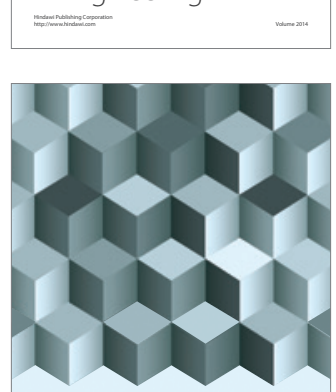

Journal of

Function Spaces
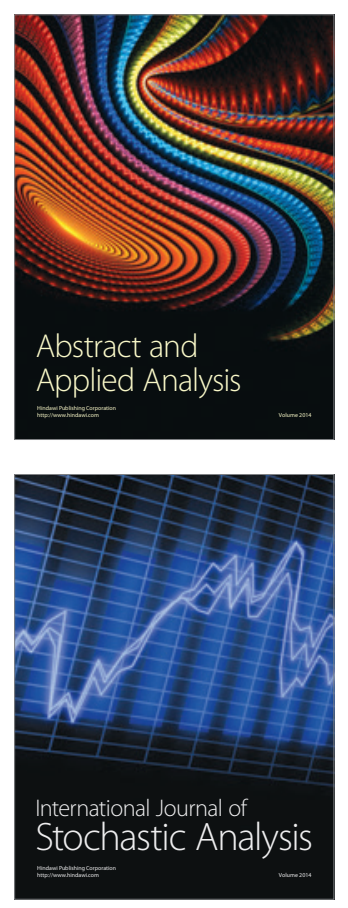

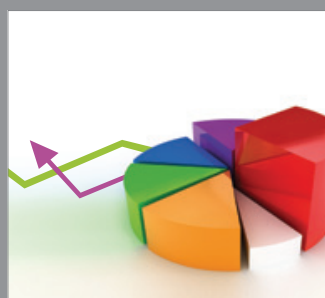

ournal of

Probability and Statistics

Promensencen
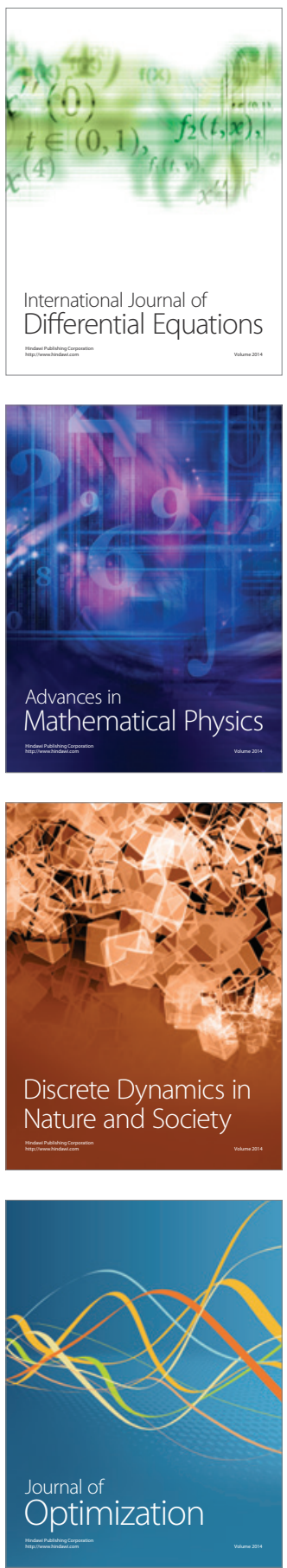